\title{
Stochastic semi-classical description of sub-barrier fusion reactions
}

\author{
Bulent Yilmaz ${ }^{1, a}$, Sakir Ayik ${ }^{2}$, and Denis Lacroix ${ }^{3}$ \\ 1 Physics Department, Ankara University, Tandogan 06100, Ankara, Turkey \\ 2 Physics Department, Tennessee Technological University, Cookeville, Tennessee 38505, USA \\ 3 GANIL, Bd Henri Becquerel, BP 55027, 14076 Caen Cedex 5, France
}

\begin{abstract}
A semi-classical method that incorporates the quantum effects of the low-lying vibrational modes is applied to fusion reactions. The quantum effect is simulated by stochastic sampling of initial zero-point fluctuations of the surface modes. In this model, dissipation of the relative energy into non-collective excitations of nuclei can be included straightforwardly. The inclusion of dissipation is shown to increase the agreement with the fusion cross section data of $\mathrm{Ni}$ isotopes.
\end{abstract}

\section{Introduction}

Sub-barrier fusion reactions are investigated by classical or semi-classical approaches known as barrier passing models [1] or fully quantum mechanical approaches such as coupled-channels model [2-4]. In fully quantal models, inclusion of the dissipation of the collective energy into intrinsic degrees of freedom and the associated fluctuations is very difficult [5-7]. However, the dissipation of relative motion can be easily incorporated in a semi-classical approximation. Recently, a stochastic semi-classical model was applied to fusion reactions of $\mathrm{Ni}$ isotopes at near-barrier energies by considering the quadrupole and octupole surface modes of both nuclei [8]. A good agreement with experimental data for fusion cross sections was obtained. The model includes coupling between the relative motion and low-lying collective surface modes of colliding nuclei. The quantum effects are included through stochastic sampling of initial zero-point fluctuations of surface vibrations. The zero-point fluctuations lead to barrier fluctuations that enhance the fusion cross-section at near and sub-barrier energies. The idea that important quantum effects can be simulated by stochastic sampling of the initial zero-point fluctuations was proposed in ref. [9]. However, only a few applications of the approach has been carried out so far [10-12].

In this contribution, we study the effect of energy dissipation due to non-collective excitations of the nuclei by using the stochastic model. The position dependent friction coefficient for the relative motion can be extracted from the linear response theory [13] and directly plugged into the equations of motion obtained by the stochastic semiclassical model. The associated fluctuations, according to the fluctuation-dissipation theorem, depend on the friction coefficient as well as on the nuclear temperature. We define the nuclear temperature via excitation energies of the surface modes. The effect of dissipation and fluctuation on the fusion cross sections is investigated. In section two, we

\footnotetext{
a e-mail: bulent.yilmaz@science.ankara.edu.tr
}

briefly introduce the model. The application of the model to fusion reactions of $\mathrm{Ni}$ isotopes is discussed in section three. The conclusion is given in section four.

\section{The Stochastic Model}

In the stochastic model, treating the surface vibrations in harmonic approximation, fusion reactions of heavy ions are described by a Hamiltonian given by

$$
\begin{aligned}
H= & \frac{P^{2}}{2 \mu}+\frac{l(l+1) \hbar^{2}}{2 \mu R^{2}}+V_{C}(R)+V_{N}\left(R, \Omega, \alpha_{i \lambda}\right) \\
& +\sum_{i=1}^{2} \sum_{\lambda=0}^{N-1}\left[\frac{\Pi_{i \lambda}^{2}}{2 D_{i \lambda}}+\frac{1}{2} C_{i \lambda} \alpha_{i \lambda}^{2}\right],
\end{aligned}
$$

where the phase space variables $(R, P)$ of the relative motion are coupled to $2 \mathrm{~N}$ vibrational modes $(\lambda=0, \ldots, N-1)$ of the fragments $(i=1,2)$ [8]. The spring constants and inertia parameters are given in terms of the deformation parameters $\beta_{i \lambda}$ and excitation energies $E_{i \lambda}^{\star}$ of the modes by $C_{i \lambda}=E_{i \lambda}^{\star} / 2 \beta_{i \lambda}^{2}$ and $D_{i \lambda}=\hbar^{2} / 2 E_{i \lambda}^{\star} \beta_{i \lambda}^{2}$, respectively [12, 14]. The first two terms of the Hamiltonian are the radial and rotational kinetic energies. $V_{C}$ is the Coulomb potential approximated as,

$$
V_{C}(R)=\left\{\begin{array}{lc}
\frac{Z_{1} Z_{2} e^{2}}{R} & R>R_{C} \\
\frac{Z_{1} Z_{2} e^{2}}{R_{C}}\left(\frac{3}{2}-\frac{1}{2} \frac{R^{2}}{R_{C}^{2}}\right) & R<R_{C}
\end{array},\right.
$$

where $R_{C}=R_{1}+R_{2}$ is the sum of the equivalent sharp radii $[15,16]$. The nuclear part of the interaction is computed using the double-folding potential as,

$$
\begin{aligned}
V_{N}\left(R, \Omega, \alpha_{i \lambda}\right)= & \int \rho_{1}\left(\mathbf{r}_{1}, \Omega_{1}, \alpha_{1 \lambda}\right) \rho_{2}\left(\mathbf{r}_{2}, \Omega_{2}, \alpha_{2 \lambda}\right) \\
& \times V_{N N}\left(\mathbf{R}-\mathbf{r}_{1}+\mathbf{r}_{2}\right) d^{3} r_{1} d^{3} r_{2} .
\end{aligned}
$$


The parameters set $\Omega=\left\{\Omega_{1}, \Omega_{2}, \Omega_{3}\right\}$ in the nuclear potential describes rotation angles of the vibration axes of the nuclei and allows to take into account all possible vibration orientations of surface modes (see ref. [8] for details). The nuclear densities $\rho_{1,2}$ are defined by two-parameter Fermi functions as

$$
\rho_{i}\left(\mathbf{r}, \alpha_{i \lambda}\right)=\frac{\rho_{0 i}}{1+\exp \left[\left(r-R_{i}\right) / a_{i}\right]},
$$

where $\rho_{0 i}$ is a normalization constant, $a_{i}$ is the diffuseness parameter and $R_{i}$ denotes the deformed nuclear radius of each nucleus. A global description of the nucleus-nucleus interaction is achieved by a zero-range nucleon-nucleon interaction of the form,

$$
V_{N N}(\mathbf{r})=V_{0} \delta(\mathbf{r})
$$

which is equivalent to a finite-range nucleon-nucleon interaction [17-20]. All the global potential and density parameters are taken from ref. [17].

The relative motion has a short de Broglie wavelength therefore the classical approximation works well for the relative motion at near and over-barrier energies where the effect of quantum tunneling is small compared to that of surface excitations. However, the dynamics of surface vibrations is far from the classical limit. In the stochastic model, the quantum effects of the surface modes are included by the initial zero-point fluctuations of the modes. A phase space distribution of the zero-point fluctuations of the surface modes can be introduced by considering the Wigner transform of the ground state which is an uncorrelated double Gaussian distribution given by

$$
F(\alpha, \Pi)=\frac{1}{2 \pi \sigma_{\alpha} \sigma_{\Pi}} \exp \left(-\frac{\alpha^{2}}{2 \sigma_{\alpha}^{2}}-\frac{\Pi^{2}}{2 \sigma_{\Pi}^{2}}\right),
$$

where $\alpha=\alpha_{i \lambda}$ and $\Pi=\Pi_{i \lambda}$. The quantal effects can be taken into account by solving the classical equations of motion given by

$$
\begin{aligned}
\frac{d R}{d t}= & \frac{P}{\mu}, \\
\frac{d P}{d t}= & -\frac{d V_{C}(R)}{d R}-\frac{\partial V_{N}\left(R, \alpha_{i \lambda}\right)}{\partial R}+\frac{l(l+1) \hbar^{2}}{\mu R^{3}} \\
& -\beta(R) P+F(t) \\
\frac{d \alpha_{i \lambda}}{d t}= & \frac{\Pi_{i \lambda}}{D_{i \lambda}}, \\
\frac{d \Pi_{i \lambda}}{d t}= & -\frac{\partial V_{N}\left(R, \alpha_{i \lambda}\right)}{\partial \alpha_{i \lambda}}-D_{i \lambda}\left(\frac{E_{i \lambda}^{\star}}{\hbar}\right)^{2} \alpha_{i \lambda} .
\end{aligned}
$$

for the initial values $\alpha_{i \lambda}^{(0)}$ and $\Pi_{i \lambda}^{(0)}$ which are randomly selected according to the Wigner distribution $F(\alpha, \Pi)$. By averaging over the resulting ensemble of events, the quantum effect of the surface vibrations is recovered. The last two terms in the right hand side of Eq.(8) are related to the energy dissipation due to coupling to intrinsic degrees of freedom. $\beta(R) P$ is the dissipation term and $F(t)$ represents the associated fluctuations. $\beta$ is the position dependent reduced friction coefficient which is calculated from the linear response theory [13] and $F(t)$, according to fluctuationdissipation theorem, is a Markovian Gaussian random number with the following correlation properties,

$$
\begin{aligned}
\langle F(t)\rangle & =0, \\
\left\langle F(t) F\left(t^{\prime}\right)\right\rangle & =2 \mu \beta k T \delta\left(t-t^{\prime}\right),
\end{aligned}
$$

where $\mu, k$, and $T$ are the reduced mass of the nuclei, Boltzmann constant, and nuclear temperature, respectively. Other than sampling of the initial values of the surface modes, $\langle\ldots\rangle$ represents an additional sampling over different values of $F(t)$.

\section{Applications}

Coupled-channels calculations are often employed for describing fusion cross-sections at sub-barrier energies. These investigations indicate that low-lying surface modes such as $2^{+}$and $3^{-}$make the dominant contribution to sub-barrier cross-sections [21-23]. Retaining only these two modes, we carry out stochastic simulations to describe the fusion process of Nickel isotopes. In order to compare our results with that of coupled-channels calculations of Nobre et al. [24], we adopt the same parameters as in that reference. It is important to note that none of these parameters are adjustable. The quadrupole $(\lambda=2)$ and octupole $(\lambda=3)$ deformation parameters are $\beta_{2}=0.215, \beta_{3}=0.263$ for ${ }^{64} \mathrm{Ni}$, and $\beta_{2}=0.205, \beta_{3}=0.235$ for ${ }^{58} \mathrm{Ni}$. The excitation energies are $E_{2}^{\star}=1.35 \mathrm{MeV}, E_{3}^{\star}=3.56 \mathrm{MeV}$ for ${ }^{64} \mathrm{Ni}$, and $E_{2}^{\star}=1.45 \mathrm{MeV}, E_{3}^{\star}=4.48 \mathrm{MeV}$ for ${ }^{58} \mathrm{Ni}$. The zerorange potential strength and the diffuseness parameter are $V_{0}=-456 \mathrm{MeVfm}^{3}$ and $a=0.56$, respectively [17].

The nuclear temperature is defined via total excitation energy of non-collective modes. The following method is used to obtain the temperature. First, the equations of motion given by Eqs. (7-10) are solved by setting $F(t)=0$. The dissipation of total energy is introduced by the noncollective friction force $-\beta(R) P$. Then, the total excitation energy of non-collective degrees of freedom is found as a function of time by using the formula,

$$
E_{\text {non-coll }}^{\star}=E_{\text {inc }}-H,
$$

where $H$ is the total Hamiltonian given by Eq. (1). Then, the temperature is found from $T=\sqrt{E_{\text {non-coll }}^{\star} / a}$ where $a$ is the level density parameter approximated as $a=A / 10$ with $A$ being the total mass number. The code is rerun by using the full dissipation and fluctuation terms $\beta$ and $F(t)$. Fig. 1 shows the total excitation energy of intrinsic degrees of freedom as a function of time for ${ }^{64} \mathrm{Ni}+{ }^{64} \mathrm{Ni}$ system at two different center of mass energies.

The fusion cross sections are calculated by using the standard equation,

$$
\sigma_{f u s}(E)=\frac{\pi \hbar^{2}}{2 \mu E} \sum_{l=0}^{l_{\max }}(2 l+1) P_{l}(E),
$$




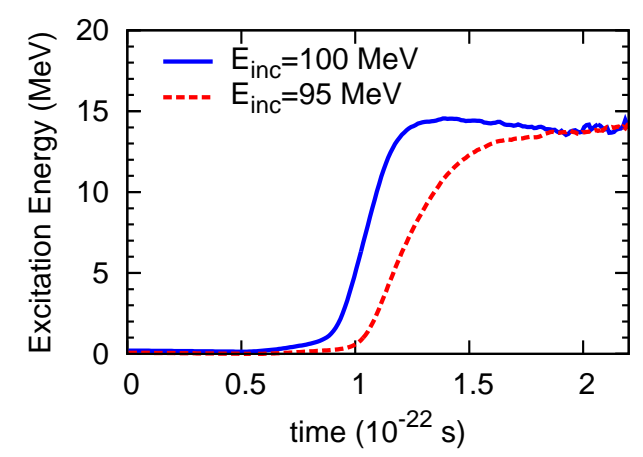

Fig. 1. The total excitation energy of the non-collective modes are plotted versus time for ${ }^{64} \mathrm{Ni}+{ }^{64} \mathrm{Ni}$ system at two different center of mass energies. The fusion barrier is $V_{B}=96.5 \mathrm{MeV}$.

where $E$ and $P_{l}(E)$ represent the initial center of mass energy and the partial transmission probabilities, respectively. The result for the fusion cross section of ${ }^{64} \mathrm{Ni}+{ }^{64} \mathrm{Ni}$ system is indicated in Fig. 2. It is seen that a good agreement with the data is obtained around the barrier where the barrier fluctuations are dominant. When the dissipation and associated fluctuation is included the agreement with the data becomes better at sub-barrier energies compared to the case without dissipation.

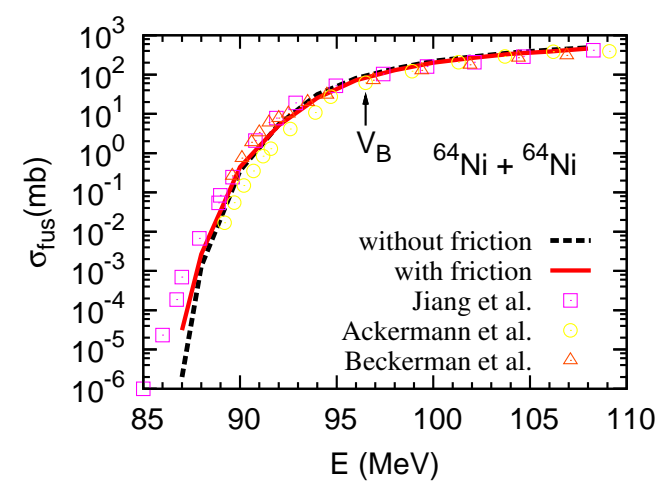

Fig. 2. The fusion cross section of ${ }^{64} \mathrm{Ni}+{ }^{64} \mathrm{Ni}$ system calculated with the stochastic zero-point model is compared with the data of ref. [25-27]. The effect of dissipation is shown. The barrier height is indicated in the figure.

In the previous work [8], some interesting observables that cannot be accessed easily by fully quantal approaches like fusion time distributions and final energy distribution of inelastic collisions were computed. The fusion time was defined as the time it takes for the relative distance to reach $5 \mathrm{fm}$ starting from $20 \mathrm{fm}$. Here, we also calculate the barrier distribution. Fig. 3 indicates the barrier distribution of ${ }^{64} \mathrm{Ni}+{ }^{64} \mathrm{Ni}$ system. Two peaks are seen in the figure which appear due to couplings to quadrupole and octupole surface modes.

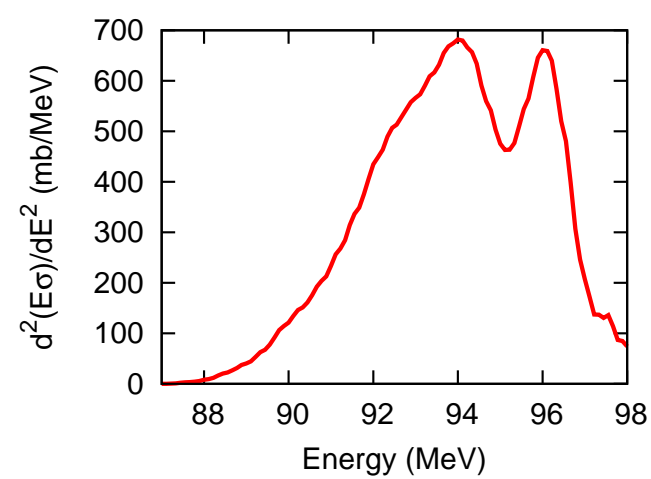

Fig. 3. The barrier distribution for ${ }^{64} \mathrm{Ni}+{ }^{64} \mathrm{Ni}$ system calculated with the stochastic zero-point model is shown.

\section{Conclusion}

Fusion reaction of ${ }^{64} \mathrm{Ni}$ ions is investigated by employing a stochastic semi-classical model which couples the relative motion to the low-frequency collective surface modes of the nuclei. The quantal effects of surface vibrations are incorporated by forming an ensemble of events of randomly selected initial values of surface modes that satisfy the corresponding Wigner distributions of the ground states. The approach is quite simple yet it can reproduce the fusion cross section data for $\mathrm{Ni}$ isotopes quite well. In the model, dissipation due to coupling to non-collective modes can be included straightforwardly. The inclusion of dissipation increases the agreement with the data. The stochastic model can also be used to obtain barrier distributions.

B.Y. and S.A. gratefully acknowledge GANIL for the support and warm hospitality extended to them during their visits. This work is supported in part by the US DOE Grant No. DE-FG0589ER40530.

\section{References}

1. A. B. Balantekin and N. Takigawa, Rev. Mod. Phys. 70, (1998) 77

2. M. J. Rhoades-Brown and M. Prakash, Phys. Rev. Lett. 53, (1984) 333.

3. S. Landowne and S. C. Pieper, Phys. Rev. C 29, (1984) 1352.

4. K. Hagino, N. Rowley, and A. T. Kruppa, Comput. Phys. Commun. 123, (1999) 143.

5. A. Diaz-Torres et al., Phys. Rev. C 78, (2008) 064604.

6. Alexis Diaz-Torres, Phys. Rev. C 82, (2010) 054617.

7. S. Yusa, K. Hagino, and N. Rowley, Phys. Rev. C 82, (2010) 024606.

8. S. Ayik, B. Yilmaz, and D. Lacroix, Phys. Rev. C 81, (2010) 034605.

9. H. Esbensen et al., Phys. Rev. Lett. 41, (1978) 296.

10. M. W. Guidry et al., Phys. Rev. C 36, (1987) 609.

11. D. Galetti et al., Phys. Rev. C 48, (1993) 3131.

12. C. H. Dasso and R. Donangelo, Phys. Lett. B 276, (1992) 1. 
13. G. G. Adamian et al., Phys. Rev. C 56, (1997) 373.

14. H. Esbensen and S. Landowne, Phys. Rev. C 35, (1987) 2090.

15. J. R. Birkelund et al., Phys. Rep. 56, (1979) 107.

16. R. W. Hasse and W. D. Myers, Geometrical Relationships of Macroscopic Nuclear Physics, (Springer-Verlag, Berlin, 1988).

17. L. C. Chamon et al., Phys. Rev. C 66, (2002) 014610.

18. M. A. C. Ribeiro et al., Phys. Rev. Lett. 78, (1997) 3270.

19. L. C. Chamon et al., Phys. Rev. Lett. 79, (1997) 5218.

20. L. R. Gasques et al., Phys. Rev. C 69, (2004) 034603.

21. H. Esbensen, Phys. Rev. C 77, (2008) 054608.

22. H. Esbensen, C. L. Jiang, and K. E. Rehm, Phys. Rev. C 57, (1998) 2401.

23. M. Beckerman, Phys. Rep. 129, (1985) 145.

24. G. P. A. Nobre et al., Nucl. Phys. A 786, (2007) 90.

25. C. L. Jiang et al., Phys. Rev. Lett. 93, (2004) 012701.

26. D. Ackermann et al., Nucl. Phys. A 609, (1996) 91.

27. M. Beckerman et al., Phys. Rev. C 25, (1982) 837. 